\title{
Clinical impact of deoxynivalenol, 3-acetyl- deoxynivalenol and 15-acetyl- deoxynivalenol on the severity of an experimental Mycoplasma hyopneumoniae infection in pigs
}

Annelies Michiels ${ }^{1 *}$ (D), loannis Arsenakis ${ }^{1}$, Anneleen Matthijs ${ }^{1}$, Filip Boyen² ${ }^{2}$, Geert Haesaert ${ }^{3}$, Kris Audenaert ${ }^{3}$, Mia Eeckhout ${ }^{3}$, Siska Croubels ${ }^{4}$, Freddy Haesebrouck ${ }^{2}$ and Dominiek Maes ${ }^{1}$

\begin{abstract}
Background: The mycotoxin deoxynivalenol (DON) is highly prevalent in cereals in moderate climates and therefore pigs are often exposed to a DON-contaminated diet. Pigs are highly susceptible to DON and intake of DONcontaminated feed may lead to an altered immune response and may influence the pathogenesis of specific bacterial diseases. Therefore, the maximum guidance level in feed is lowest in this species and has been set at $900 \mu \mathrm{g} / \mathrm{kg}$ feed by the European Commission. This study aimed to determine the effect of in-feed administration of a moderately high DON concentration $(1514 \mu \mathrm{g} / \mathrm{kg})$ on the severity of an experimental Mycoplasma hyopneumoniae (M. hyopneumoniae) infection in weaned piglets. Fifty M. hyopneumoniae-free piglets were assigned at 30 days of age [study day (D)0] to four different groups: 1) negative control group (NCG; $n=5), 2)$ DON-contaminated group (DON; $n=15), 3)$ DON-contaminated and M. hyopneumoniae-inoculated group (DONMHYO; $n=15)$, 4) M. hyopneumoniae-inoculated group (MHYO; $n=15)$. The piglets were fed the experimental diets ad libitum for five weeks and were monitored during this period and euthanized at day 35 [27 days post infection (DPI)] or 36 (28 DPI). The main parameters under investigation were macroscopic lung lesions (MLL) at euthanasia, respiratory disease score (RDS) from day 8 until day 35, histopathologic lesions and log copies of M. hyopneumoniae DNA detected by qPCR, determined at the day of euthanasia.
\end{abstract}

Results: No significant difference was obtained for MLL at euthanasia, RDS (8-35), histopathologic lung lesions and log copies of M. hyopneumoniae DNA in the DONMHYO and MHYO group and consequently, no enhancement of the severity of the M. hyopneumoniae infection could be detected in the DONMHYO compared to the MHYO group.

Conclusions: Under present conditions, the findings imply that feed contaminated with DON $(1514 \mu \mathrm{g} / \mathrm{kg})$ provided to weaned pigs for five weeks did not increase the severity of an experimental M. hyopneumoniae infection. Further research is needed to investigate the impact of DON on M. hyopneumoniae infections in a multi-mycotoxin and multi-pathogen environment.

Keywords: Mycoplasma hyopneumoniae, Experimental, Challenge, Clinical, Impact, Deoxynivalenol, Feed, Weaned piglets

\footnotetext{
* Correspondence: Annelies.Michiels@UGent.be

1Department of Reproduction, Obstetrics and Herd Health, Faculty of

Veterinary Medicine, Ghent University, Salisburylaan 133, 9820 Merelbeke,

Belgium

Full list of author information is available at the end of the article
}

(C) The Author(s). 2018 Open Access This article is distributed under the terms of the Creative Commons Attribution 4.0 International License (http://creativecommons.org/licenses/by/4.0/), which permits unrestricted use, distribution, and reproduction in any medium, provided you give appropriate credit to the original author(s) and the source, provide a link to the Creative Commons license, and indicate if changes were made. The Creative Commons Public Domain Dedication waiver (http://creativecommons.org/publicdomain/zero/1.0/) applies to the data made available in this article, unless otherwise stated. 


\section{Background}

The mycotoxin deoxynivalenol (DON) is a fungal metabolite produced mainly by Fusarium graminearum and Fusarium culmorum [1, 2]. Fusarium-produced mycotoxins, of which by toxicological viewpoint the trichothecenes DON and T-2 toxin, zearalenone and fumonisins are the most important [3], have been reported worldwide in many cereal-based cropping systems. Fusarium species have traditionally been associated with temperate cereals, as these fungi require lower temperatures for growth and mycotoxin production [4]. Indeed, DON is one of the most common natural mycotoxin contaminants of wheat and other small cereal grains harvested in moderate climate zones [5-7]. Extensive data on global mycotoxin occurrence showed that $59 \%$ of 5819 samples of animal feed tested positive on DON presence $[3,4,8]$. In low doses, DON causes anorexia, decreased weight gain and immune stimulation [2]. Pigs are known to be a very sensitive animal species to DON, mainly because of the high oral bioavailability and differences in metabolism of this mycotoxin compared to other species [2]. In moderate to high doses (above $840 \mu \mathrm{g} / \mathrm{kg}$ feed), decreased feed intake or feed refusal, vomiting and immune suppression are seen $[2,9,10]$. In fact, the EU (European Union) recommended maximum pig feed guidance level for DON is $900 \mu \mathrm{g} / \mathrm{kg}$, which is the lowest one compared to other farm animal species for total diets and compounds of total diets. For adult ruminants and poultry a maximum of $5000 \mu \mathrm{g} /$ $\mathrm{kg}$ and $2000 \mu \mathrm{g} / \mathrm{kg}$ for calves and lambs are set as guidance levels [11]. On top of that, the pig consumes a cereal rich diet and DON is frequently detected in wheat, barley, corn and by-products $[9,12]$.

It is known that DON can have an impact on the pathogenesis of several bacterial diseases $[3,13,14]$. Exposing porcine ileal loop tissue to a DON-concentration of $1 \mu \mathrm{g} / \mathrm{ml}$, potentiated the inflammatory response and significantly enhanced Salmonella Typhimurium invasion in and passage of the bacterium across the intestinal epithelium [15]. Furthermore, DON $(0.025 \mu \mathrm{g} / \mathrm{ml})$ induced an enhanced uptake of Salmonella Typhimurium in porcine macrophages, indicating the capacity of DON to modulate the innate immune system, and thus to increase the susceptibility of the pig to Salmonella Typhimurium infections [16]. Deoxynivalenol might, due to the immunomodulatory effect on the host and/or the immediate impact on the pathogen, have an impact on the course of respiratory infectious diseases in swine. However only limited in vivo information is available. A three-week ingestion period of feed contaminated with high levels of $2500 \mu \mathrm{g} / \mathrm{kg}$ and $3500 \mu \mathrm{g} / \mathrm{kg}$ DON resulted in a higher viremia and lung viral load in case of a
Porcine Circovirus type 2 (PCV2) infection, and a lower body weight gain, more lung lesions and mortality in porcine reproductive and respiratory syndrome virus (PRRSv)-infected pigs, respectively [17, 18]. Pigs receiving fumonisin B1 in a concentration of $10,000 \mu \mathrm{g} / \mathrm{kg}$ feed, and dually infected with Bordetella bronchiseptica (B. bronchiseptica) and Pasteurella multocida, (P. multocida) were at greater risk to develop pneumonia and had an increase of the extent and severity of the pathological changes compared to dually infected pigs that did not receive fumonisin B1 [19]. Oral gavage of $P$. multoci$d a$-infected pigs with a crude extract of fumonisin B1 in a concentration of $500 \mu \mathrm{g} / \mathrm{kg}$ body weight (BW) per day for a period of seven days resulted in the pigs coughing more, in increased bronchoalveolar lavage fluid total cells, macrophages and lymphocytes, and resulted in an increased occurrence of lung lesions compared to the pigs only infected with P. multocida [20]. Dietary exposure to fumonisin B1 in a concentration of $12,000 \mu / \mathrm{kg}$ feed increased the risk on PRRSv-associated disease [21] and induced pulmonary edema which may aggravate $M$. hyopneumoniae infection [22].

Mycoplasma hyopneumoniae is causing tremendous economic losses in all intensive pig producing countries worldwide [23], despite many attempts to control the disease (enzootic pneumonia) through vaccination strategies and control measures. Consequently, there is a high prevalence of both $M$. hyopneumoniae infections and a high contamination rate of feed with mycotoxins, more specific DON, in Europe [8]. Therefore, the odds for a pig to ingest feed contaminated with DON, whilst simultaneously being infected with $M$. hyopneumoniae is high. The present study aimed to investigate the effect of in-feed administration of DON at a moderately high level of $1540 \mu \mathrm{g} / \mathrm{kg}$ feed, on the clinical course of an experimental $M$. hyopneumoniae infection with two genetically different $M$. hyopneumoniae strains in weaned piglets.

\section{Methods}

\section{Study animals and experimental design}

The study was compliant with all relevant institutional and European standards for animal care and experimentation. The experiment was approved by the Ethics Committee for Animal Experiments of the Faculty of Veterinary Medicine and Faculty of Bioscience Engineering, Ghent University (approval number EC2015/112). Fifty M. hyopneumoniae-free Rattlerow-Seghers piglets (RA-SE Genetics NV, Ooigem, Belgium) were included in the study. The herd of origin has been free of M. hyopneumoniae and PRRSv since 2012 based on repeated serological testing, absence of clinical signs and pneumonia lesions, and nested polymerase chain reaction (nPCR) testing on tracheobronchial swabs as previously described [24]. The gilts and sows in the herd were vaccinated 
against Erysipelothrix rhusiopathiae and Parvovirus before insemination. No vaccinations were administered to the piglets. The piglets were weaned on average at 26 days of age and moved four days later to the experimental facilities of the Faculty of Veterinary Medicine, Ghent University, Belgium. The piglets were individually identified by means of an ear tag. The study design, the different parameters and timing are summarized in Table 1. Upon arrival (D0) the piglets were randomly allocated to four different groups: 1) negative control group (NCG; $n=5)$ : sham-inoculated D8, D9 + control diet, 2) DON-contaminated group (DON; $n=$ 15): sham-inoculated D8, D9 and DON-diet (1514 $\mu \mathrm{g} / \mathrm{kg}), 3$ ) DON-contaminated and $M$. hyopneumoniae-inoculated group (DONMHYO; $\mathrm{n}=15$ ): experimentally inoculated with M. hyopneumoniae D8, D9 and DON-diet (1514 $\mu \mathrm{g} / \mathrm{kg}), 4$ ) M. hyopneumoniae-inoculated group (MHYO; $\mathrm{n}=15)$ : experimentally inoculated with $M$. hyopneumoniae D8, D9+ control diet. The number of 15 animals in the treatment groups enabled to find a difference of $5.26 \pm 4.7$ in the main parameter, namely macroscopic lung lesions (two-sided test) with $95 \%$ certainty and a statistical power of $80 \%$. This difference is biologically relevant and was based on previous research in our research group [25]. The NCG (5 pigs) was used to verify whether the purchased piglets remained $M$. hyopneumoniae negative throughout the study. The different groups were housed in four different facilities equipped with absolute filtered chambers (HEPA U15) in order to avoid cross-infection of $M$. hyopneumoniae between the different groups. The pigs had free access to drinking water and were fed ad libitum.

\section{Mycoplasma hyopneumoniae strains and challenge infection}

The pigs were inoculated with two different strains of M. hyopneumoniae: a highly virulent strain F7.2C and low virulent strain F1.12. Both strains had been differentiated and characterized at proteomic level with Sodium-Dodecyl-Sulphate Polyacrylamide gelelectrophoresis (SDS-page) [26] and at genomic level with Random Amplified Polymorphic DNA (RAPD), Amplified Fragment Length Polymorphism (AFLP), PCR-Random Fragment Length Polymorphism (PCR-RFLP) of the p146 gene, Variable Number of Tandem Repeats (VNTR) analysis of p97, with Multiple-Locus of VNTR Analysis (MLVA) [27-29] and used in previous studies [24, 25, 30]. Previous research has shown that pigs are often infected with two or even three genetically different $M$. hyopneumoniae strains [29, 31, 32], and in slaughter pigs infected with different strains, more lung lesions can be detected [32]. Therefore, the pigs in the present study were inoculated with two genetically different $M$. hyopneumoniae strains, as performed previously by this research group [18], to mimic the situation in the field. All pigs were anesthetised via the intramuscular route with $0.22 \mathrm{ml} / \mathrm{kg}$ BW of a mixture of tiletamine, zolazepam (Zoletil $100^{\circ}$, Virbac, Louvain-la-Neuve, Belgium) and xylazine (Xyl-M ${ }^{\circ}$

Table 1 Experimental design, sample collections and timing in the different experimental groups

\begin{tabular}{|c|c|c|c|c|}
\hline \multirow{2}{*}{$\begin{array}{l}\text { Study day, } \\
\text { D }\end{array}$} & \multicolumn{4}{|l|}{ Groups } \\
\hline & NCG $(n=5)$ & $\mathrm{DON}(n=15)$ & DONMHYO $(n=15)$ & $\mathrm{MHYO}(n=15)$ \\
\hline \multirow[t]{3}{*}{$\overline{\mathrm{DO} 0^{\mathrm{a}}}$} & Arrival & & & \\
\hline & Weight & & & \\
\hline & Randomisation & & & \\
\hline D0-D35/36 & Commercial feed ad libitum & DON-contaminated feed ad libitum & DON-contaminated feed ad libitum & Commercial feed ad libitum \\
\hline$D 1-D 35^{c}$ & RDS & & & \\
\hline \multirow[t]{3}{*}{ D8 } & Sham-inoculation ${ }^{d}$ & Sham-inoculation ${ }^{d}$ & F7.2C $\mathrm{C}^{\mathrm{e}}$ inoculation & F7.2C $\mathrm{C}^{\mathrm{e}}$-inoculation \\
\hline & Weight & & & \\
\hline & Blood & & & \\
\hline D9 & Sham-inoculation ${ }^{d}$ & Sham-inoculation ${ }^{d}$ & F1.12A-inoculation & F1.12A-inoculation \\
\hline \multirow[t]{2}{*}{ D21 } & BALF & & & \\
\hline & Blood & & & \\
\hline \multirow[t]{4}{*}{ D35/36 } & Weight & & & \\
\hline & Necropsy & & & \\
\hline & Lung sample & & & \\
\hline & BALF & & & \\
\hline
\end{tabular}

athe average age of the pigs at arrival was 26 days, ${ }^{b}$ all pigs of the NCG and DON group, and five pigs of MHYO were necropsied at D35. Ten pigs of MHYO and all pigs of DONMHYO were necropsied at D36, ${ }^{\mathrm{C}} \mathrm{RDS}$ was not determined at D0 and D36 because the piglets arrived later than 8 a.m. (hour of performing coughing score every day) and part of the piglets were already euthanized on D36, respectively, ${ }^{d}$ sham-inoculation was performed with sterile Friis medium, ehighly virulent strain of $M$. hyopneumoniae, flow virulent strain of $M$. hyopneumoniae

NCG negative control group, DON deoxynivalenol contaminated group, DONMHYO deoxynivalenol contaminated $+M$. hyopneumoniae-inoculated group, MHYO M. hyopneumoniae-inoculated group, RDS respiratory disease score, BALF bronchoalveolar lavage fluid 
2\%, VMD, Arendonk, Belgium) and the pigs of the DONMHYO and MHYO groups were endotracheally inoculated with $7 \mathrm{ml}$ of inoculum containing $10^{7} \mathrm{CCU} / \mathrm{ml}$ of strain $\mathrm{F} 7.2 \mathrm{C}$ on $\mathrm{D} 8$ and $7 \mathrm{ml}$ of inoculum containing $10^{7} \mathrm{CCU} / \mathrm{ml}$ of strain F1.12A on D9. On both inoculation days, the pigs of the NCG and DON group were endotracheally sham-inoculated with $7 \mathrm{ml}$ of sterile Friis medium. At D35 or D36 of the experiment, the pigs were euthanized using deep anaesthesia by intramuscularly administering $0.3 \mathrm{ml} / \mathrm{kg} \mathrm{BW}$ of a mixture of tiletamine, zolazepam (Zoletil $100^{\circ}$, Virbac, Louvain-la-Neuve, Belgium) and xylazine (Xyl-M $\mathrm{M}^{\bullet} \%$, VMD, Arendonk, Belgium), followed by exsanguination. For practical reasons and to avoid $M$. hyopneumoniae contamination of the samples, all pigs of the NCG and DON group were euthanized at D35, followed by five animals of the MHYO group. All the other animals of the MHYO and all animals of the DONMMHYO group were necropsied on D36.

\section{Deoxynivalenol contaminated diet}

A commercial antibiotic-free diet for weaned piglets was purchased (Leievoeders N.V., Waregem, Belgium). Before purchasing the feed, a sample of the batch was tested with liquid chromatography-tandem mass spectrometry (LC-MS/MS) according to Monbaliu et al. [33, 34] for the presence of DON, 3-Acetyldeoxynivalenol (3-ADON), zearalenone and fumonisin $\mathrm{B} 1+\mathrm{B} 2$, the levels were below the reporting limit of $50 \mu \mathrm{g} / \mathrm{kg}, 50 \mu \mathrm{g} / \mathrm{kg}, 10 \mu \mathrm{g} / \mathrm{kg}$ and $50 \mu \mathrm{g} / \mathrm{kg}$, respectively. The piglets of the NCG and MHYO groups were fed this commercial diet from D0 until D35/ 36. A part of the purchased feed $(1300 \mathrm{~kg})$ was transported to the laboratory of the Department of Applied Biosciences (Faculty of Bioscience Engineering, Ghent University) to add the target concentration of $1800 \mu \mathrm{g} / \mathrm{kg}$ feed DON. This procedure was followed: the reference strain Fusarium graminearum (F. graminearum) MUCL 42841 (Mycothèque de l'Université catholique de Louvain) was used to produce the DON-culture. The strain was grown in liquid mineral (MIN) medium supplemented with L-arginin as a selective nitrogen source, as previously described by Gardiner et al. [35]. After 14 days of cultivation, the culture was filtered and centrifuged. The obtained concentration of DON was determined with LC-MS/MS by adding $150 \mu \mathrm{l}$ of the resulting undiluted MIN medium to $5 \mathrm{~g}$ of certified blank wheat standard (Sigma Aldrich, Overijse, Belgium). In total, $7535 \mathrm{mg} / \mathrm{kg}$ DON was quantified and $1076 \mathrm{mg} / \mathrm{kg}$ acetylated DON (3-ADON + 15-ADON) in the grown DON-culture. Next to the presence of DON, the inoculum was tested with LC-MS/MS for the presence of other F. graminearum trichothecenes such as nivalenol, neosolaniol, fusarenon-X, diacetoxyscirpenol, HT-2 toxin and $\mathrm{T}-2$ toxin, and results were below the detection limit. Also no zearalenone was detected. Eight 1 of inoculum were obtained for preparation of $1300 \mathrm{~kg}$ of
DON-contaminated feed in a concentration aimed at twice the recommended maximum pig feed level of $900 \mu \mathrm{g} / \mathrm{kg}$ or $1800 \mu \mathrm{g} / \mathrm{kg}$ DON. First, $2.67 \mathrm{l}$ of the inoculum was mixed with $10 \mathrm{~kg}$ of feed to obtain a thoroughly mixed premix of the DON-contaminated feed. Subsequently, the premix with inoculum was added to $433.3 \mathrm{~kg}$ of feed in a feed mill and thoroughly mixed for at least $40 \mathrm{~min}$. The same procedure was repeated twice, to obtain the total amount of $1300 \mathrm{~kg}$ of DON-contaminated feed in a concentration of $1800 \mu \mathrm{g} / \mathrm{kg}$ and the feed was collected again in the original $25 \mathrm{~kg}$ bags of the feeding company. After preparation of the contaminated feed, a mixed sample originating from three DON-contaminated feed bags was taken and was submitted for LC-MS/MS to obtain the true DON-concentration of the contaminated feed. The results of the LC-MS/MS of the contaminated feed were $407 \pm$ $120 \mu \mathrm{g} / \mathrm{kg}$ DON, $280 \pm 100 \mu \mathrm{g} / \mathrm{kg} 3-\mathrm{ADON}$ and $827 \pm$ $300 \mu \mathrm{g} / \mathrm{kg} 15-\mathrm{ADON}$, resulting in a total DON and acetylated $\mathrm{DON}$ (3-ADON + 15-ADON)-concentration of $1514 \mu \mathrm{g} / \mathrm{kg}$ in the contaminated feed sample. This feed was used in the DON and DONMHYO-groups from D0 until D35/36 of the study.

\section{Clinical and performance parameters}

From D0 until D35/36 onwards, the piglets were observed daily at $8 \mathrm{a} . \mathrm{m}$. for at least half an hour by the same researcher to assess appetite, faecal consistency and presence of dyspnea and tachypnea. A faecal consistency score was used to evaluate the faeces found on the pen floor before cleaning [36]: 1 (firm and shaped), 2 (soft and shaped), both addressed as a normal faecal consistency in pigs, 3 (loose) and 4 (watery), with scores 3 and 4 considered as abnormal. Daily, from D1 until D35 a respiratory disease score (RDS), ranging from 0 to 6 was recorded according to Halbur et al. (1996) [37]. Score 0 was obtained when a pig did not cough. Score 1, 3 and 5 were respectively designated as mild, moderate and severe couging after encouraged move. Score 2, 4, 6 were respectively obtained when mild, moderate and severe coughing in rest was present.The RDS was not determined at D0 and D36, as the pigs arrived later than 8 a.m. at the facilities and already part of the animals was euthanized on D35. The daily RDS values were averaged for the following periods: D1-7, D8-35/36 and D1-35/36. All pigs were weighed $(\mathrm{kg})$ at the day of arrival (D0), the first inoculation day (D8) and the day of euthanasia (D35/36). The average daily gain (ADG, $\mathrm{kg} / \mathrm{pig} /$ day) was calculated from D0-7, D8-35/36 and D0-35/36 by subtracting the starting weights from the final weights, divided by the number of days during that period.

\section{Macroscopic and histopathologic lung lesions}

The lungs were removed and macroscopic lung lesions (MLL) (D35/36) were determined according to Hannan 
et al. (1982) [38] from each pig. Consequently, the lungs were transported to the laboratory of the Department of Pathology, Bacteriology and Avian Diseases, Faculty of Veterinary Medicine, Ghent University from the necropsy rooms from the experimental facilities in the same department and from each lung in each pig, samples from the right apical, cardiac and diaphragmatic lung lobes were collected. In case a lesion was present, a sample was collected including both healthy and affected lung tissue, at the border of the lesion. The $10 \%$ neutral formalin fixed and paraffin embedded samples were stained with hematoxylin and eosin. Using light microscopy the samples were investigated and scored for the degree of peribronchiolar and perivascular lymphohistiocytic infiltration and nodule formation (cuffing) [39]. The scoring system ranged from 1 to 5 , with score 1 and 2 considered not to be related with $M$. hyopneumoniae infection as previously described [24, 39, 40].The percentage of air (percentage of lung area occupied by air) was examined by means of an automated image analysis system (Leica application suite AF Lite (Diegem, Belgium) and image J (Bethesda, Maryland, USA) [41]. This parameter is inversely proportional to the lymphohistiocytic infiltration in the lung tissue and the intrabronchiolar-and bronchial exudate [25].

\section{Quantitative PCR for M. hyopneumoniae}

Two weeks post inoculation (PI) (D21) of the high virulent strain F7.2C (D8), bronchoalveolar lavage (BAL) fluid from all pigs, while conscious was collected. After snaring the pigs and opening their mouth with a gag, a catheter (Portex ${ }^{\circ}$ Dog Catheter with Female Luer Mount, Smiths Medical International Ltd. Kent, United Kingdom) was inserted allowing to flush the lungs with $10 \mathrm{ml}$ of sterile phosphate buffered saline (PBS). The PBS fluid was subsequently aspirated. At the day of necropsy (D35/36) the head bronchus of the left part of the lung was flushed with $10 \mathrm{ml}$ of sterile PBS before collection of the histopathological samples. After collection, the BAL fluids were stored at $-70{ }^{\circ} \mathrm{C}$ until they were analysed. The DNA was extracted with the DNeasy Blood \& Tissue kit (QIAGEN, Qiagen Benelux, B.V., Antwerp, Belgium) with the DNA Purification protocol for bloods or bloody fluids (spin protocol) on $200 \mu \mathrm{l}$ of BAL fluid according to the manual instructions and quantitative PCR (qPCR) was performed as previously described to detect the number of $M$. hyopneumoniae organisms [42]. Briefly, after DNA-extraction, qPCR was performed with primers p102f (5'GTCAAAGTCAAAGT CAGCAAAC 3') and p102r (5'AGCTGTTCAAATGC TTGTCC 3') using SensiMixTM SYBR (Bioline GmbH, Luckenwalde, Germany) in the CFX384 real-time PCR detection system (Bio-Rad, Nazareth, Belgium). A tenfold dilution series of M. hyopneumoniae DNA of strain
F7.2C was used to convert the threshold values to the number of M. hyopneumoniae organisms. Values below the dilution of $1.50 \times 10^{1}$ (1.18 $\log$ copies) were considered as negative [31, 42].

\section{Serology}

A blocking ELISA (IDEIA ${ }^{\text {mo }}$ Mycoplasma hyopneumoniae EIA kit, Oxoid Limited, Hampshire, UK) was performed according to the instructions in the protocol manual and as previously described [24] to detect antibodies against M. hyopneumoniae in the blood collected at D28, D21 and at necropsy (D35/36). Sera with optical density< $50 \%$ of the average value of the OD-buffer control were considered to be positive. All values above or equal to $50 \%$ of the average value of the OD-buffer control were classified as negative .

\section{Routine bacteriological culture on bronchoalveolar lavage fluid}

Ten $\mu \mathrm{l}$ of BAL fluid collected at necropsy (D35/36) of each pig was inoculated on Columbia agar supplemented with 5\% sheep blood (Oxoid, Hampshire, UK) with a Staphylococcus pseudintermedius streak for bacteriological examination [43]. Plates were incubated for $48 \mathrm{~h}$ in a $5 \% \mathrm{CO}_{2}$-enriched environment at $35{ }^{\circ} \mathrm{C}$ for identification of respiratory bacteria in the lungs. All macroscopically different colonies were identified to the species level (score value $>2.000$ ) with a Bruker Daltonic Microflex LT Biotyper Biotyper MALDI-TOF mass spectrometer by using the direct transfer method and $\alpha$-cyano-4-hydroxycinnamic acid (HCCA) as matrix, according to the manufacturer's guidelines. The spectra were obtained and analysed with the MBT Compass software version 3.1. (Bruker Daltonik), which included a database of 6903 mean spectra projections.

\section{Statistical analysis}

The independent variable in the statistical analyses was 'group': NCG, DON, DONMHYO and MHYO-group. The dependent variables were RDS, weight, ADG, MLL, histopathology and percentage of air, qPCR-results, percentage of $M$. hyopneumoniae qPCR-positive samples, $M$. hyopneumoniae specific $\mathrm{AB}$ expressed in $\mathrm{OD}$ values and percentage of ELISA M. hyopneumoniae positive samples. These variables were all run in separate models with 'pig' as statistical unit and no additional factors included into the model. The normality of the data was investigated by means of descriptive statistics, except for the binary data (M. hyopneumoniae $\mathrm{qPCR}$ positive samples and ELISA M. hyopneumoniae positive samples). The parameters BW, ADG and percentage air analysis were normally distributed and a one-way analysis of variance (ANOVA) test was used. In case of the RDS, a repeated measures ANOVA was performed. Scheffés 
post-hoc test was used to make pair-wise comparisons. The qPCR-results and M. hyopneumoniae specific antibodies were not normally distributed and therefore, a non-parametric Kruskal-Wallis test was used, with the Dunn-Bonferroni approach to make pair-wised comparisons, as well for MLL and histopathology results. In case of the normally distributed data, the mean and standard deviation (SD) were reported, in case of the non-parametric data, the median and the interquartile range were reported. All analyses for these parameters were performed with SPSS 23 for Windows (SPSS inc. Illinois, USA). Percentage of seropositive pigs and percentage of pigs testing positive with qPCR in each group were analyzed using binomial logistic regression ( $R$ version 3.3.1) [44]. The results were considered to be statistically significant when $P<0.05$.

\section{Results}

\section{Clinical and performance parameters}

In none of the groups, feed refusals or vomiting were observed. No tachypnea, nor dyspnea were observed in any of the groups. Post-weaning diarrhoea was observed from D0 onwards until D3 in all groups, therefore all pigs were treated IM with Colistin sulphate (Colivet 'S', Prodivet,
Eynatten, Belgium) from D0 onwards for 5 days, according to the product leaflet. The post weaning diarrhoea lasted until D8, D3, D3 and D7 in NCG, DON, DONMHYO and $\mathrm{MHYO}$, respectively. In three groups some pigs with faecal consistency score 3 were noticed throughout the study: in NCG two pigs at D20 and D23 respectively, in DON one pig at D13 and in DONMHYO two pigs at D20 and $\mathrm{D} 23$, respectively. In MHYO, normal faecal consistency was observed throughout the study. Coughing was not observed in the NCG. All results of the different time periods for this parameter in the study are shown in Table 2 (RDS $_{1-7}, \mathrm{RDS}_{8-35}$ and RDS ${ }_{1-35}$ ). The RDS from the first inoculation day onwards until euthanasia (RDS ${ }_{8-35}$ ) were $0 \pm 0, \quad 0.0071 \pm 0.028, \quad 1.04 \pm 0.82,1.14 \pm 0.92$ for NCG, DON, DONMHYO and MHYO, respectively $(P<0.001)$. No statistically significant differences were obtained between all groups for D1-7. For D8-35 and D1-35 a statistically significant difference was obtained between the experimentally infected (DONMHYO and MHYO) and non-infected pigs (NCG and DON) $(P<0.001)$. However, no statistically significant differences were obtained for DONMHYO and MHYO groups in each time period of the study (Table 2). The daily course of RDS ${ }_{1-35}$ for each group is shown in Fig. 1.

Table 2 Results of the clinical parameters, macroscopic and microscopic lung lesions in the different experimental groups

\begin{tabular}{|c|c|c|c|c|c|}
\hline \multirow[t]{2}{*}{ Parameter } & \multicolumn{5}{|l|}{ Groups } \\
\hline & NCG $(n=5)$ & DON $(n=15)$ & DONMHYO $(n=15)$ & $\mathrm{MHYO}(n=15)$ & $P$-value \\
\hline \multicolumn{6}{|l|}{$\overline{\mathrm{RDS}}$} \\
\hline D1-7 & $0 \pm 0$ & $0 \pm 0$ & $0 \pm 0$ & $0 \pm 0$ & 1.00 \\
\hline D8-35 & $0 \pm 0^{\mathrm{a}}$ & $0.01 \pm 0.03^{\mathrm{a}}$ & $1.04 \pm 0.82^{b}$ & $1.14 \pm 0.92^{b}$ & $<0.001$ \\
\hline D1-35 & $0 \pm 0^{\mathrm{a}}$ & $0.01 \pm 0.03^{\mathrm{a}}$ & $0.83 \pm 0.84^{b}$ & $0.91 \pm 0.94^{b}$ & $<0.001$ \\
\hline \multicolumn{6}{|l|}{ Weight \pm SD (kg) } \\
\hline D0 & $6.37 \pm 1.09$ & $6.39 \pm 0.92$ & $6.38 \pm 1.04$ & $6.42 \pm 1.23$ & 1.00 \\
\hline D8 & $7.70 \pm 1.13$ & $8.04 \pm 1.27$ & $8.20 \pm 1.08$ & $8.11 \pm 1.90$ & 0.92 \\
\hline D35/36 & $20.10 \pm 2.33$ & $19.45 \pm 3.83$ & $21.02 \pm 3.18$ & $21.75 \pm 4.36$ & 0.38 \\
\hline \multicolumn{6}{|l|}{ ADG (kg/pig/day) } \\
\hline D0-8 & $0.52 \pm 0.14$ & $0.53 \pm 0.19$ & $0.56 \pm 0.15$ & $0.67 \pm 0.17$ & 0.12 \\
\hline D0-35/36 & $0.39 \pm 0.06$ & $0.37 \pm 0.11$ & $0.41 \pm 0.08$ & $0.43 \pm 0.10$ & 0.41 \\
\hline D8-35/36 & $0.46 \pm 0.08$ & $0.42 \pm 0.13$ & $0.46 \pm 0.10$ & $0.49 \pm 0.10$ & 0.37 \\
\hline \multicolumn{6}{|c|}{ MLL, histopathology and percentage of air D35/36 } \\
\hline MLL & $0 \pm 0^{a}$ & $0 \pm 0^{a}$ & $2.77 \pm 3.22^{b}$ & $5.87 \pm 7.32^{b}$ & $<0.001$ \\
\hline Histopathology & $1.70 \pm 0.20^{\mathrm{a}}$ & $2.00 \pm 0.30^{\mathrm{a}}$ & $2.40 \pm 0.80^{b}$ & $2.40 \pm 0.90^{b}$ & $<0.001$ \\
\hline Percentage of air (\%) & $45.94 \pm 6.54$ & $46.85 \pm 6.86$ & $49.75 \pm 9.90$ & $46.96 \pm 8.51$ & 0.25 \\
\hline
\end{tabular}

Respiratory disease score (RDS), bodyweight, average daily weight gain (ADG), macroscopic lung lesions (MLL), histopathology score of the lungs and percentage of air in the lungs for NCG, DON group, DONMHYO group, MHYO group

The parameters bodyweight, ADG, percentage of air analysis were analysed by means of one way analysis of variance, the parameter RDS with a repeated measures analysis of variance, in both cases with Scheffé post-hoc test to make pair-wised comparisons, therefore means \pm SD are reported. The parameter MLL and histopathology were analysed with Kruskal-Wallis test and the Dunn-Bonferroni approach to make pair-wised comparison, therefore medians and interquartile range are reported. Different superscripts in one row are statistically different $(P<0.05)$

NCG: negative control group, DON: deoxynivalenol contaminated group, DONMHYO: deoxynivalenol contaminated $+M$. hyopneumoniae-inoculated group, MHYO: $M$. hyopneumoniae-inoculated group, $S D$ : standard deviation, $n$ number, $D$ Day of the study, $A D G$ average daily gain, RDS respiratory disease score, $M L L$ macroscopic lung lesions 
There were no significant differences between the groups for the parameter BW and the ADG during the different time periods (Table 2).

\section{Macroscopic and histopathologic lung lesions}

The MLL of the NCG, DON, DONMHYO and MHYO groups were $0 \pm 0,0 \pm 0,2.77 \pm 3.22$ and 5.87 \pm 7.32 , respectively $(P<0.001)$. The histopathological lung lesions were $1.70 \pm 0.20,2.00 \pm 0.30,2.40 \pm 0.80$ and $2.40 \pm 0.90$ for the NCG, DON, DONMHYO and MHYO group, respectively $(P<0.001)$. The percentage of air was $45.94 \pm 6.54,46.85 \pm 6.86,49.75 \pm 9.90$ and $46.96 \pm 8.51$ for NCG, DON, DONMHYO and MHYO, respectively $(P=0.25)$. There were no statistically significant differences between the DONMHYO and MHYO groups for these above-mentioned parameters, however there were statistically significant differences between the experimentally infected (DONMHYO and MHYO) and non-infected pigs (NCG and DON) $(P<0.001)$, except for the parameter percentage of air analysis $(P=0.25)$ (Table 2 ).

\section{Quantitative PCR for M. hyopneumoniae}

The samples of the NCG and DON group remained negative throughout the study. The qPCR results at D21 were $0.05 \pm 0.37,0.63 \pm 0.81,3.92 \pm 2.60$ and 3.24 $\pm 1.91(P<0.001)$ and at $\mathrm{D} 35 / 360.80 \pm 0.70,0.29 \pm$ $1.09,4.05 \pm 1.38$ and $4.20 \pm 1.00(P<0.001)$ for NCG,
DON, DONMHYO and MHYO, respectively. There were no significant differences between the DONM$\mathrm{HYO}$ and MHYO group, however there were statistically significant differences between the experimentally infected (DONMHYO and MHYO) and non-infected pigs (NCG and DON) $(P<0.001)$ for D21 and D35/36 (Table 3$)$.

\section{Serology}

The serological results are presented in Table 3. All pigs of the NCG and DON remained serologically negative throughout the study. The OD-values of the serological results at $\mathrm{D} 35 / 36$ were $1.15 \pm 0.27,1.16 \pm 0.18,0.25 \pm$ 0.18 and $0.23 \pm 0.09$ for the NCG, DON, DONMHYO and MHYO, respectively. At necropsy (D35/36), all pigs of DONMHYO and MHYO were serologically positive.

\section{Routine bacteriological culture on bronchoalveolar lavage fluid}

Few colonies of Streptococcus suis were isolated from one pig of both the DONMHYO (1/15) and MHYO (1/ $15)$ groups. In addition, few colonies of $B$. bronchiseptica were isolated from one pig of DON (1/15) and four pigs of MHYO (4/15).

\section{Discussion}

In the present study, the outcome of the main parameters (RDS, MLL, histopathological lesions and log copies of M. hyopneumoniae DNA in bronchoalveolar lavage

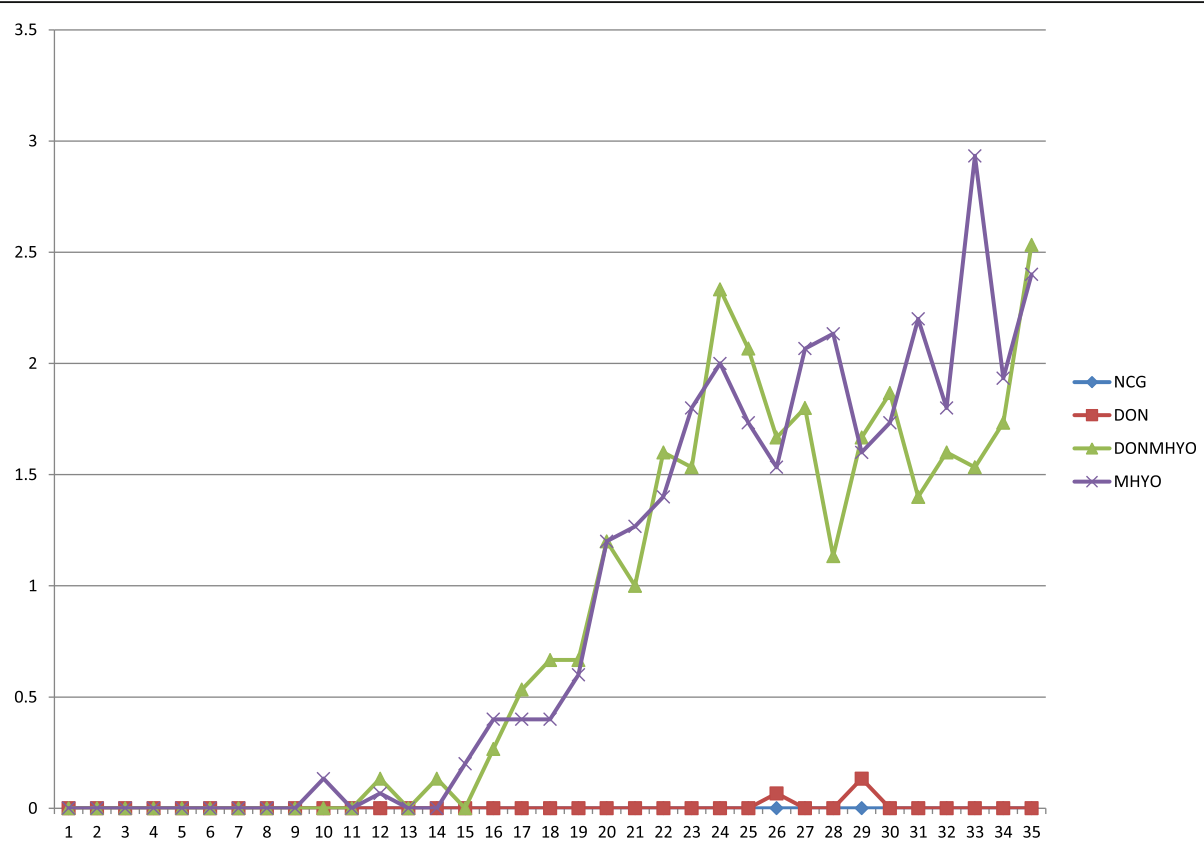

Fig. 1 Course of average respiratory disease score (RDS) from day 1 until the day of necropsy (D 35). Average RDS from D1 until D35 in the negative control group (NCG), the DON-contaminated group (DON), DON-contaminated and M. hyopneumoniae-inoculated group (DONMHYO), and M. hyopneumoniae-inoculated group (MHYO). The challenge infections were performed on D8 (a highly virulent M. hyopneumoniae strain F7.2C) and D9 (a low virulent strain F1.12) in DONMHYO and MHYO. The NCG and DON were sham-inoculated with sterile Friis medium on D8 and D9 
Table 3 Results of M. hyopneumoniae-DNA detection in the BALF and serology in the different experimental groups

\begin{tabular}{|c|c|c|c|c|c|}
\hline \multirow[t]{2}{*}{ Parameter } & \multicolumn{5}{|l|}{ Groups } \\
\hline & NCG $(n=5)$ & $\mathrm{DON}(n=15)$ & DONMHYO $(n=15)$ & $\mathrm{MHYO}(n=15)$ & $P$-value \\
\hline \multicolumn{6}{|c|}{ qPCR (log copies of M. hyopneumoniae DNA /ml BALF) \pm SD } \\
\hline D21 & $0.05 \pm 0.37^{\mathrm{a}}$ & $0.63 \pm 0.81^{\mathrm{a}}$ & $3.92 \pm 2.60^{b}$ & $3.24 \pm 1.91^{\mathrm{b}}$ & $<0.001$ \\
\hline D35/36 & $0.80 \pm 0.70^{\mathrm{a}}$ & $0.29 \pm 1.09^{\mathrm{a}}$ & $4.05 \pm 1.38^{\mathrm{b}}$ & $4.20 \pm 1.00^{\mathrm{b}}$ & $<0.001$ \\
\hline \multicolumn{6}{|c|}{ Percentage of M. hyopneumoniae aPCR positive samples ( $\mathrm{n}$ of positive samples/total $\mathrm{n}$ of samples tested) } \\
\hline D21 (\%) & $0(0 / 5)^{a}$ & $0(0 / 15)^{a}$ & $87(13 / 15)^{b}$ & $93(14 / 15)^{b}$ & $<0.001$ \\
\hline D35/36 (\%) & $0(0 / 5)^{a}$ & $0(0 / 15)^{a}$ & $100(15 / 15)^{\mathrm{b}}$ & $100(15 / 15)^{b}$ & $<0.001$ \\
\hline \multicolumn{6}{|c|}{ M. hyopneumoniae specific $A B$ expressed in $\mathrm{OD}$-values $\pm \mathrm{SD}$} \\
\hline D8 & $1.68 \pm 0.23$ & $1.83 \pm 0.34$ & $1.71 \pm 0.19$ & $1.70 \pm 0.19$ & 0.27 \\
\hline D21 & $1.15 \pm 0.22^{\mathrm{a}}$ & $1.29 \pm 0.23^{\mathrm{a}}$ & $1.16 \pm 0.36^{\mathrm{ab}}$ & $0.94 \pm 0.26^{\mathrm{b}}$ & 0.001 \\
\hline D35/36 & $1.15 \pm 0.27^{\mathrm{a}}$ & $1.16 \pm 0.18^{\mathrm{a}}$ & $0.25 \pm 0.18^{b}$ & $0.23 \pm 0.10^{b}$ & $<0.001$ \\
\hline \multicolumn{6}{|c|}{ Percentage of ELISA M. hyopneumoniae positive samples ( $n$ of positive samples/total $n$ of samples tested) } \\
\hline D8 (\%) & $0(0 / 5)$ & $0(0 / 15)$ & $0(0 / 15)$ & $0(0 / 15)$ & 1.00 \\
\hline D21 (\%) & $0(0 / 5)$ & $0(0 / 15)$ & $0(0 / 15)$ & $13(2 / 15)$ & 0.17 \\
\hline $\mathrm{D} 35 / 36(\%)$ & $0(0 / 5)^{a}$ & $0(0 / 15)^{a}$ & $100(15 / 15)^{b}$ & $100(15 / 15)^{b}$ & $<0.001$ \\
\hline
\end{tabular}

Respiratory disease score (RDS), bodyweight, average daily weight gain (ADG), macroscopic lung lesions (MLL), histopathology score of the lungs and percentage of air in the lungs for NCG, DON group, DONMHYO group, MHYO group

The parameter $\mathrm{PPCR}$ and $M$. hyopneumoniae specific $\mathrm{AB}$ were analysed with a non-parametric Kruskal-Wallis and the Dunn-Bonferroni approach to make pair-wised comparisons, therefore median and interquartile range are reported. The prevalence of $M$. hyopneumoniae qPCR positive samples and the prevalence of ELISA M. hyopneumoniae positive samples were analysed with binomial logistic regression. Different superscripts in one row are statistically different $(P<0.05)$

NCG: negative control group, DON: deoxynivalenol contaminated group, DONMHYO: deoxynivalenol contaminated + M. hyopneumoniae-inoculated group, MHYO: $M$. hyopneumoniae-inoculated group, Different superscripts in one row are statistically different $(P<0.05), n$ number, $S D$ standard deviation, $D$ Day of the study, $M$. hyopneumoniae: Mycoplasma hyopneumoniae, $A B$ antibodies, $O D$ optical densities

fluid) was not statistically different in M. hyopneumoniae infected pigs that received or did not receive DON-contaminated feed. This indicates that ingestion of DON-contaminated feed at a concentration of $1514 \mu \mathrm{g} /$ $\mathrm{kg}$, exceeding the maximum guidance level for pigs according to EU regulation, did not aggravate the severity of an experimental $M$. hyopneumoniae-infection in weaned piglets.

The challenge infection was successful as all animals in the M. hyopneumoniae inoculated groups (MHYO and DONMHYO group) coughed, showed lung lesions (except for one animal in the MHYO group), seroconverted and $M$. hyopneumoniae-DNA was detected in BAL fluid at necropsy. The results obtained in the M. hyopneumoniae challenged pigs (DONMHYO and MHYO group) differed significantly from the non-challenged pigs (DON group, NCG). The latter pigs remained serologically negative throughout the entire study period and no M. hyopneumoniae- DNA was detected in BAL fluid two weeks post-inoculation, nor at necropsy. The obtained data in the challenged groups were comparable with a previous study performed by our research group with the same challenge model [24], demonstrating the repeatability of the model. The two $M$. hyopneumoniae-strains were not administered on the same day, as the infection model practiced at our research group uses seven $\mathrm{ml}$ inoculum containing $10^{7}$
$\mathrm{CCU}$ of the strain/ml [31]. It is not known whether piglets of four to five weeks of age (on average 26 days, D0 at arrival and inoculation at D8) are able to cope with twice the inoculum volume (14 $\mathrm{ml}$ for both strains). In addition, it is not known whether both strains will grow next to each other, or whether one strain will overgrow the other strain consuming most of the nutrients, when these $M$. hyopneumoniae-strains are grown in the same culture flask. Consequently, it was decided to challenge the piglets on two consecutive days.

In the present study, DON was aimed to be administered at two times the maximum guidance level of DON advised by the European commission for pigs $(1800 \mu \mathrm{g} /$ $\mathrm{kg}$ feed) [11]. This dose was selected as Madson et al. [45] stated that moderate $(1000-5000 \mu \mathrm{g} / \mathrm{kg}$ feed) to high concentrations ( $>5000 \mu \mathrm{g} / \mathrm{kg}$ feed) are associated with delayed or supressed immune responses due to leukocyte apoptosis and resulting in increased disease susceptibility $[45,46]$. It was demonstrated that a dose-related decrease in daily feed intake is observed when administering DON-supplemented feed to pigs, and that this effect was mostly significant to the control feed in the range of $2000 \mu \mathrm{g} / \mathrm{kg}-4000 \mu \mathrm{g} / \mathrm{kg}$ [47-49]. Therefore, a dose below this range was chosen to be administered to the pigs in this study to limit feed refusals and to avoid hampering of DON possibly influencing the investigated parameters. The commercial, un-spiked feed 
was tested before the start of the study, not only for the presence of DON, but also for zearalenone and fumoni$\sin \mathrm{B} 1+\mathrm{B} 2$, to avoid these components having an effect in the feed of the control groups (MHYO group, NCG) and avoiding an additional or synergistic effect to the DON-toxicity in the DON-spiked groups (DON group, DONMHYO group) [46]. After preparation of the contaminated feed, a mixed feed sample from three DON-contaminated feed bags was submitted to LC-MS/ MS to detect the DON-concentration and to ensure thorough mixing of the DON-inoculum in the feed. The results of the LC-MS/MS was $1514 \mu \mathrm{g} / \mathrm{kg}$ of DON and acetylated forms and deviated only slightly from the target concentration of $1800 \mu \mathrm{g} / \mathrm{kg}$. Cytotoxicity of the produced DON in the study was not tested on beforehand, as the deleterious effects of DON in the pigs are known and the same DON inoculation method in pig feed was already successfully used in a study by Goossens et al. [50]. In the latter study, the in vivo effect of DON on intestinal damage influencing the resorption of doxycycline was determined in pigs.

It remains to be elucidated why no impact of DON in the pigs was observed in the present study. The degree of susceptibility of certain breeds/lines to the effects of DON might be one explanation [51]. For instance a lower severity in porcine circovirus type 2 associated histopathological lesions has been shown for Piétrain compared to landrace pigs [52], but so far no studies have assessed the impact of genetic differences in sensitivity to the effects of DON.

No feed-refusals were observed in the pigs administered the DON-contaminated feed. The minimum emetic dose for orally distributed DON in pigs is $100 \mu \mathrm{g} / \mathrm{kg}$ [53], yet vomiting was not observed in this study. The reason why this did not occur is unclear. It must be noted that the observation period of the pigs (30 min. of RDS-scoring, 15 min. Feeding and cleaning in the morning, $10 \mathrm{~min}$. of observation and cleaning in the evening) was fairly short compared to the time the pigs spent in the facilities. However, the main researcher was always present after providing the feed to identify possible sick pigs, as most healthy pigs start eating immediately after providing the feed and Young et al., [54] observed that vomiting occurs within minutes after ingesting the DON-contaminated feed. Pestka et al. [53] stated that vomiting due to DON-contaminated feed, is more likely if the feed is ingested at once and not via smaller portions throughout the day. The contaminated feed and hence the DON in the DONMHYO and DON pigs, was consumed throughout the day as the pigs were fed ad libitum and had freely access to the feed.

The M. hyopneumoniae-infected pigs administered the DON-supplemented feed did not have a higher RDS compared to the pigs only infected with $M$. hyopneumoniae.
No other studies are available investigating the impact of DON on respiratory tract disease signs in M. hyopneumoniae-infected pigs. However the effect of fumonisin B1 in combination with $M$. hyopneumoniae or the effect of DON on other pathogens has been studied. Pósa et al., [22] studied the effect of $20,000 \mu \mathrm{g} / \mathrm{kg}$ fumonisin B1 on M. hyopneumoniae-infected pigs, however no firm conclusions could be drawn regarding the difference in coughing between the $M$. hyopneumoniae-infected pigs with or without fumonisin B1 supplementation in the feed. Halloy et al. [20] investigated the impact of fumonisin B1 administered orally $(500 \mu \mathrm{g} / \mathrm{kg} \mathrm{BW}$ per day, seven days) in $P$. multocida-infected pigs and concluded that these pigs coughed more compared to the pigs only infected with $P$. multocida.

It is known that $M$. hyopneumoniae infections can negatively influence production parameters, such as ADG [55]. Deoxynivalenol ingestion in pigs may result in reduced feed intake, and subsequently decrease ADG [51, 56, 57]. In the present study, DON did not decrease ADG in the $M$. hyopneumoniae inoculated animals. This result is in agreement with Accensi et al. [9] (840 $\mu \mathrm{g}$ DON/kg feed), Gerez et al. [46] (1500 $\mu \mathrm{g}$ DON $/ \mathrm{kg}$ feed), and Savard et al. [17]. The latter authors studied the impact of DON (2500 and $3000 \mu \mathrm{g}$ DON $/ \mathrm{kg}$ feed) in pigs that were simultaneously infected with PCV2. Pósa et al., [22], did not find an effect of fumonisin B1 $(20,000 \mu \mathrm{g} / \mathrm{kg})$ on ADG in $M$. hyopneumoniae-infected pigs. Rotter et al. [58] observed an adaptation of pigs to oral ingestion of Fusarium mycotoxins from one week onwards. In that study, pigs were fed a diet mixed with naturally contaminated corn $(28,700 \mu \mathrm{g} / \mathrm{kg}$ DON, $8600 \mu \mathrm{g} / \mathrm{kg} 15-\mathrm{ADON}$ and1,100 $\mu \mathrm{g} /$ $\mathrm{kg}$ ZEA) to obtain DON-concentrations of 750,1500 or $3000 \mu \mathrm{g}$ DON $/ \mathrm{kg}$ feed during 28 days. During the first week, the exposed pigs had lower weight gains than the pigs fed a non-contaminated control feed. At the end of the study, however, the overall weight gains did not differ anymore between these groups. This adaptation might be one of the reasons why we did no see an effect of DON on daily growth. The relative low number of pigs, followed up during a limited period of time, which is inherent in experimental infection studies like ours, also makes it difficult to obtain a statistical significant difference in daily growth $[31,59,60]$. No effect of DON ingestion on the macroscopic and histopathological lung lesions was observed in the M. hyopneumoniae infected group. This finding is in agreement with Pósa et al. [19], who neither saw a statistical difference in macroscopic lung lesions between the $B$. bronchiseptica and $P$. multocida dually-infected groups with or without supplementation of fumonisin B1 in the feed $(10,000 \mu \mathrm{g} / \mathrm{kg})$ of three-day-old piglets. Savard et al. (2015) on the other hand, did observe an effect of DON-supplementation in the feed $(3500 \mu \mathrm{g} / \mathrm{kg}$, three weeks) on macroscopic lung 
lesions in PRRSv-infected pigs [51]. In this study, DON-ingestion did not influence the number of log copies detected in the M. hyopneumoniae infected animals. Similarly, Savard et al. [61] did not observe an effect of DON-contamination on the presence of viral RNA, measured with qPCR, in PRRSv-infected pigs. It is not clear why the obtained effects of dietary DON vary among experiments, It might be explained by different factors such as starting weight or age of the pigs, the contamination source of DON (natural versus artificial contamination), presence of other known or unknown undetected fungal metabolites or pathogens, duration of the study (adaptation), number of pigs used in the study, gender of the pigs, health status, nutritional balance of the pig and statistical design of the study $[10,62,63]$. It is not known why DON did not influence a $M$. hyopneumoniae infection under the circumstances in this study. Deoxynivalenol has a good distribution in the lung of the pig [64]. However, as $M$. hyopneumoniae is attached to the cilia of the upper respiratory tract and does not invade the parenchyma of the lung $[23,65,66]$, it might be that DON is not able to exert its effect on the pathogen. More research is needed to investigate this relationship in pigs, for instance by in vitro tests on pig tracheal explants. On the other hand discrepancies have been reported between in vivo (higher viremia in pigs exposed to DON in the feed) and in vitro (decreased PRRSv replication in MARC-cells) effects of DON on PRRSv $[18,61]$, thus extrapolation of in vitro results to in vivo effects has to be done cautiously.

\section{Conclusions}

No effect was observed of DON contamination in a moderately high dose in the feed on the severity of an experimental M. hyopneumoniae infection in weaned piglets. In the field, however, the impact of DON-contaminated feed on a $M$. hyopneumoniae-infection might be more expressed, because mostly multi-mycotoxin contamination of the feed occurs $[3,52-54]$, the pigs can be exposed to DON-contaminated feed for a longer period than the five week exposure period in this study, often suboptimal housing and climate conditions may prevail and other pathogens may be present $[55,56]$. Further research should assess the impact of DON on M. hyopneumoniae infections under these multi-pathogen and multi-mycotoxins circumstances and investigating the impact of DON in vitro on M. hyopneumoniae in tracheal explant cells. More research could also focus on factors influencing the effect on DON such as health status, gender, age, and possible genetic resistance of the pigs.

\section{Abbreviations}

3-ADON: 3-Acetyldeoxynivalenol; ADG: average daily gain; ANOVA: one way analysis of variance; BAL: bronchoalveolar lavage; BW: body weight; D: study day; DON: deoxynivalenol; DON: DON-contaminated; DONMHYO: DONcontaminated and M. hyopneumoniae- inoculated; EU: European Union; F. graminearum: Fusarium graminearum; LC-MS/MS: liquid chromatographytandem mass spectrometry; MHYO: M. hyopneumoniae- inoculated; MIN: liquid mineral; MLL: macroscopic lung lesions; NCG: negative control group; $\mathrm{nPCR}$ : nested polymerase chain reaction; PBS: phosphate buffered saline; PI: post inoculation; qPCR: quantitative PCR; RDS: respiratory disease score

\section{Acknowledgements}

The authors are grateful to Marleen Foubert for the assistance with preparing the inoculum, to Pieter-Jan De Temmerman for the help with the binary data analysis and to the colleagues for assistance during the necropsy Thank you to the feeding company Leievoeders N.V. for the flexibility in re-testing and delivering the feed and thank you to RA-SE for wanting to provide the $M$. hyopneumoniae-negative pigs.

\section{Funding}

The study was partially financed by ZOETIS, Belgium. The role of the funding body was merely financial. ZOETIS, Belgium did not have any role in the design of the study, collection, analysis of the data, and writing of the manuscript.

\section{Availability of data and materials}

The datasets used and/or analyzed during the current study are available from the corresponding author on reasonable request.

\section{Authors' contributions}

AMi designed the study protocol, wrote the ethical application, grew the $M$. hyopneumoniae strains for the experimental infections, mixed the feed with the DON-inoculum, performed the trial, performed the laboratory analyses, performed the statistical analyses, interpreted the data and wrote the manuscript. IA reviewed the study protocol, assisted with performing the trial, assisted with interpretation of the data and reviewed the manuscript. AMa reviewed the study protocol, assisted with performing the trial, assisted with the lab work, assisted with interpretation of the data and reviewed the manuscript. FB reviewed the study protocol, the ethical application dossier, assisted with interpretation of the data and reviewed the manuscript and helped with the laboratory analysis. $\mathrm{GH}$ and KA were responsible for producing the DON inoculum from the reference strain of $F$. graminearum and supervised the production of the DON-contaminated feed. ME and SC assisted with the interpretation of the mycotoxin testing of the commercial feed and the DON-inoculated feed. GH, KA, ME, SC, FH and DM designed the study protocol, reviewed the ethical application dossier and the manuscript and assisted with interpretation of the data. All authors gave their final approval for publication of the study.

\section{Ethics approval and consent to participate}

The study was compliant with all relevant institutional and European standards for animal care and experimentation. The experiment was approved by the Ethics Committee for Animal Experiments of the Faculty of Veterinary Medicine and Faculty of Bioscience Engineering, Ghent University (approval number EC2015/112). Consent to participate: not applicable.

Competing interests

The authors declare that they have no competing interests.

\section{Publisher's Note}

Springer Nature remains neutral with regard to jurisdictional claims in published maps and institutional affiliations.

\section{Author details}

'Department of Reproduction, Obstetrics and Herd Health, Faculty of Veterinary Medicine, Ghent University, Salisburylaan 133, 9820 Merelbeke, Belgium. ${ }^{2}$ Department of Pathology, Bacteriology and Avian Diseases, Faculty of Veterinary Medicine, Ghent University, Salisburylaan 133, 9820 Merelbeke, Belgium. ${ }^{3}$ Department of Applied Biosciences, Faculty of Bioscience Engineering, Ghent University, Campus Schoonmeersen, Valentin Vaerwyckweg 1, 9000 Ghent, Belgium. ${ }^{4}$ Department of Pharmacology, Toxicology and Biochemistry, Faculty of Veterinary Medicine, Ghent University, Salisburylaan 133, 9820 Merelbeke, Belgium. 


\section{Received: 22 December 2017 Accepted: 24 May 2018}

Published online: 18 June 2018

\section{References}

1. Whitlow WM, Hagler JR, Diaz DE. Mycotoxins in feed. Feedstuffs. 2010:74-84.

2. Bracarense AP, Lucioli J, Grenier B, Drociunas Pacheco G, Moll WD, Schatzmayr $\mathrm{G}$, et al. Chronic ingestion of deoxynivalenol and fumonisin, alone or in interaction, induces morphological and immunological changes in the intestine of piglets. Br J Nutr. 2012:107:1776-86.

3. Antonissen G, Martel A, Pasmans F, Ducatelle R, Verbrugghe E, Vandenbroucke $V$, et al. The impact of Fusarium mycotoxins on human and animal host susceptibility to infectious diseases. Toxins. 2014;6:430-52.

4. Placinta CM, D'Mello JPF, Macdonald AMC. A review of worldwide contamination of cereal grains and animal feed with Fusarium mycotoxins. Anim Feed Sci Technol. 1999:78:21-37.

5. Kostelanska M, Dzuman Z, Malachova A, Capouchova I, Prokinova E, Skerikova A, et al. Effects of milling and baking technologies on levels of deoxynivalenol and its masked form deoxynivalenol-3-glucoside. J Agric Food Chem. 2011;59:9303-12.

6. Miller JD. Mycotoxins in small grains and maize: old problems, new challenges. Food Addit Contam. 2008;25:219-30.

7. Rasmussen PH, Nielsen KF, Ghorbani F, Spliid NH, Nielsen GC, Jørgensen LN. Occurrence of different trichothecenes and deoxynivalenol-3-B-D-glucoside in naturally and artificially contaminated Danish cereal grains and whole maize plants. Mycotoxin Res. 2012;28:181-90.

8. Rodrigues I, Naehrer K. A three-year survey on the worldwide occurrence of mycotoxins in feedstuffs and feed. Toxins. 2012;4:663-75.

9. Accensi F, Pinton P, Callu P, Abella-Bourges N, Guelfi J-F, Grosjean F, Oswald IP. Ingestion of low doses of deoxynivalenol does not affect hematological, biochemical, or immune responses of piglets. J Anim Sci. 2006;84:1935-42.

10. Dersjant-Li Y, Verstegen MW, Gerrits WJ. The impact of low concentrations of aflatoxin, deoxynivalenol or fumonisin in diets on growing pigs and poultry. Nutrition Res Rev. 2003;16:223-39.

11. Commission recommendation of 17 August 2006 on the presence of deoxynivalenol, zearalenone, ochratoxin A, T-2, HT-2 and fumonisin in products intended for animal feeding. In: Ojeu. vol. L229;2006;7-9.

12. Pierron A, Alassane-Kpembi I, Oswald I. Impact of mycotoxin on immune response and consequences for pig health. Anim Nutr. 2016;2:63-8.

13. Antonissen G, Van Immerseel F, Pasmans F, Ducatelle R, Haesebrouck F, Timbermont $L$, et al. The mycotoxin deoxynivalenol predisposes for the development of Clostridium perfringens-induced necrotic enteritis in broilers. PLoS One. 2014;9:1-8.

14. Antonissen G, Croubels S, Pasmans F, Ducatelle R, Eeckhaut V, Devreese M, et al. Fumonisins affect the intestinal microbial homeostasis in broiler chickens. predisposing to necrotic enteritis Vet Res. 2015;46:1-11.

15. Vandenbroucke V, Croubels S, Martel A, Verbrugghe E, Goossens J, Van Deun $\mathrm{K}$, et al. The mycotoxin Deoxynivalenol potentiates intestinal inflammation by Salmonella Typhimurium in porcine lleal loops. PLoS One. 2011;6:1-8.

16. Vandenbroucke V, Croubels S, Verbrugghe E, Boyen F, De Backer P, Ducatelle R, et al. The mycotoxin deoxynivalenol promotes uptake of Salmonella Typhimurium in porcine macrophages, associated with ERK1/2 induced cytoskeleton reorganization. Vet Res. 2009:40:1-12.

17. Savard C, Provost C, Alvarez F, Pinilla V, Music N, Jacques M, et al. Effect of deoxynivalenol (DON) mycotoxin on in vivo and in vitro porcine circovirus type 2 infections. Vet Microbiol. 2015;176:257-67.

18. Savard C, Pinilla V, Provost C, Gagnon CA, Chorfi Y. In vivo effect of deoxynivalenol (DON) naturally contaminated feed on porcine reproductive and respiratory syndrome virus (PRRSV infection. Vet Microbiol. 2014;147:419-26.

19. Pósa R, Donkó T, Bogner P, Kovács M, Repa I, Magyar T. Interaction of Bordetella bronchiseptica, Pasteurella multocida, and fumonisin B1 in the porcine respiratory tract as studied by computed tomography. Can J Vet Res. 2011;75:176-82.

20. Halloy DJ, Gustin PG, Bouhet S, Oswald I. Oral exposure to culture material extract containing fumonisins predisposes swine to the development of pneumonitiscaused by Pasteurella multocida. Toxicology. 2005;213:34-44.

21. Ramos CM, Martinez EM, Carrasco AC, Puente JHL, Quezada F, Perez JT, et al. Experimental trial of the effect of Fumonisin B1 and the PRRS virus in swine. J Anim Vet Adv. 2010;9:1301-10.

22. Pósa R, Magyar T, Stoev SD, Glávits R, Donkó T, Repa I, et al. Use of computed tomography and histopathologic review for lung lesions produced by the interaction between Mycoplasma hyopneumoniae and Fumonisin mycotoxins in pigs. Vet Pathol. 2013;50:971-9.

23. Maes $D$, Sibila M, Kuhnert $P$, Segalés J, Haesebrouck F, et al. Update on Mycoplasma hyopneumoniae infections in pigs: knowledge gaps for improved disease control. Transbound Emerg Dis. 2017;1:1-15.

24. Michiels A, Arsenakis I, Boyen F, Krecjci R, Haesebrouck F, Maes D. Efficacy of one dose vaccination against experimental infection with two Mycoplasma hyopneumoniae strains. BMC Vet Res. 2017;13:1-10.

25. Vicca J. 2005. Virulence and antimicrobial susceptibility of Mycoplasma hyopneumoniae isolates from pigs. PhD thesis. Faculty of Veterinary Medicine, Ghent University, ISBN 90-5864-086-8, 219 pp.

26. Calus D, Baele M, Meyns T, de Kruif A, Butaye P, Decostere A, et al. Protein variability among Mycoplasma hyopneumoniae isolates. Vet Microbiol. 2007; 120:284-91.

27. Stakenborg T, Vicca J, Butaye P, Maes D, Peeters J, de Kruif A, et al. The diversity of Mycoplasma hyopneumoniae within and between herds using pulsed-field gel electrophoresis. Vet Microbiol. 2005;109:29-35.

28. Stakenborg T, Vicca J, Maes D, Peeters J, de Kruif A, Haesebrouck F, et al. Comparison of molecular techniques for the typing of Mycoplasma hyopneumoniae isolates. J Microbiol Methods. 2006;66:263-75.

29. Vranckx K, Maes D, Calus D, Villarreal I, Pasmans F, Haesebrouck F. Multiple locus variable number of tandem repeats analysis is a suitable tool for the differentiation of Mycoplasma hyopneumoniae strains without cultivation. J Clin Microbiol. 2011:49:2020-3.

30. Villarreal I, Maes D, Meyns T, Gebruers F, Calus D, Pasmans F, et al. Infection with a low virulent Mycoplasma hyopneumoniae isolate does not protect piglets against subsequent infection with a highly virulent $M$. hyopneumoniae isolate. Vaccine. 2009;27:1875-9.

31. Vranckx K, Maes D, Del Pozo Sacristán R, Pasmans F. Haesebrouck F. A longitudinal study of the diversity and dynamics of Mycoplasma hyopneumoniae infections in pig herds. Vet Microbiol. 2011;156:315-21.

32. Michiels A, Vranckx K, Piepers S, Del Pozo Sacristán R, Arsenakis I, Boyen F, et al. Impact of diversity of Mycoplasma hyopneumoniae strains on lung lesions in slaughter pigs. Vet Res. 2017:48:1-14.

33. Monbaliu S, Van Poucke C, Van Peteghem C, Van Poucke K, Heungens K, De Saeger $\mathrm{S}$. Development of a multi-mycotoxin liquid chromatography/ tandem mass spectrometry method for sweet pepper analysis. Rapid Commun Mass Spectrom. 2009;23:3-11.

34. Monbaliu S, Van Poucke C, DeTavernier C, Dumoulin F, Van De Velde M, Schoeters $\mathrm{E}$, et al. Occurrence of mycotoxins in feed as analyzed by a multimycotoxin LC-MS/MS method. J Agric Food Chem. 2010;58:66-71.

35. Gardiner DM, Kazan K, Manners JM. Nutrient profiling reveals potent inducers of trichothecene biosynthesis in fusarium graminearum. Fungal Genet Biol. 2009:46:604-13.

36. Pedersen KS, Toft N. Intra- and inter-observer agreement when using a descriptive classification scale for clinical assessment of faecal consistency in growing pigs. Prev Vet Med. 2011;98:288-91.

37. Halbur PG, Paul PS, Meng XJ, Lum MA, Andrews JJ, Rathje JA. Comparative pathogenicity of nine US porcine reproductive and respiratory syndrome virus (PRRSV) isolates in a five-week-old cesarean-derived, colostrumdeprived pig model. J Vet Diagn Investig. 1996;8:11-20.

38. Hannan PC, Bhogal BS, Fish JP. Tylosin tartrate and tiamutilin effects on experimental piglet pneumonia induced with pneumonic pig lung homogenate containing mycoplasmas, bacteria and viruses. Res Vet Sci 1982;33:76-88.

39. Morris C, Gardner I, Hietala S, Carpenter T, Anderson R, Parker K. Seroepidemiologic study of natural transmission of Mycoplasma hyopneumoniae in a swine herd. Prev Vet Med. 1995;21:323-37.

40. Del Pozo Sacristán R. Treatment and control of Mycoplasma hyopneumoniae infections. Ghent: Ghent University; 2014.

41. Rasband WS. ImageJ In.: U. S. National Institutes of Health, Bethesda, Maryland, USA; 1997-2016.

42. Marois C, Dory D, Fablet C, Madec F, Kobisch M. Development of a quantitative real-time TaqMan PCR assay for determination of the minimal dose of Mycoplasma hyopneumoniae strain 116 required to induce pneumonia in SPF pigs. J Appl Microbiol. 2010;108:1523-33.

43. Villarreal I, Maes D, Vranckx K, Calus D, Pasmans F, Haesebrouck F. Effect of vaccination of pigs against experimental infection with high and low virulence Mycoplasma hyopneumoniae strains. Vaccine. 2011;29:1731-5.

44. R: A language and environment for statistical Computing URL https://www. R-project.org/, Accessed 18 Nov 2017. 
45. Madson DM, Ensley SM, Patience JF, Gauger PC, Main RG. Diagnostic assessment and lesion evaluation of chronic deoxynivalenol ingestion in growing swine. Swine Health Prod. 2014;22:78-83.

46. Pestka JJ. Mechanisms of deoxynivalenol-induced gene expression and apoptosis. Food Addit Contam Part A. 2008;25:1128-40.

47. Bergsjø B, Matre T, Nafstad I. Effects of diets with graded levels of deoxynivalenol on performance in growing pigs. Zentralbl Veterinarmed $A$. 1992;39:752-8.

48. Dänicke S, Goyaerts T, Valenta H, Razzazi E, Böhm J. On the effects of deoxynivalenol (DON) in pig feed on growth performance, nutrients utilization and DON metabolism. J Anim Feed Sci. 2004;13:539-56.

49. Prelusky DB, Gerdes RG, Underhill KL, Rotter BA, Jui PY, Trenholm HL. Effects of low-level dietary deoxynivalenol on haematological and clinical parameters of the pig. Nat Toxins. 1994;2:97-104.

50. Goossens J, Vandenbroucke V, Pasmans F, De Baere S, Devreese M, Osselaere A, et al. Influence of mycotoxins and a mycotoxin adsorbing agent on the oral bioavailability of commonly used antibiotics in pigs. Toxins. 2012;4:281-95.

51. Eriksen GS, Pettersson $\mathrm{H}$. Toxicological evaluation of trichothecenes in animal feed. Anim Feed Sci Technol. 2004;114:205-39.

52. Opriessnig T, Patterson AR, Madson DM, Pal N. Difference in severity of porcine circovirus type two-induced pathological lesions between landrace and Pietrain pigs. J Anim Sci. 2009;87:1582-90.

53. Pestka JJ. Deoxynivalenol: toxicity, mechanisms and animal health risks. Anim Feed Sci Technol. 2007;137:283-98.

54. Young LG, McGirr L, Valli VE, Lumsden JH, Lun A. Vomitoxin in maize fed to young pigs. J Anim Sci. 1983;57:655-64.

55. Thacker EL, Minion FC. Mycoplasmosis. In: Zimmerman JJ, Karriker LA, Ramirez A, Schwartz KJ, Stevenson GW, editors. Diseases of Swine, 9th edition. Ames, lowa: Wiley-Blackwell;2012.

56. Friend DW, Trenholm HL, Elliot JI, Thompson BK, Hartin KE. Effect of feeding vomitoxin-contaminated wheat to pigs. Can J Anim Sci. 1982;62:1211-22.

57. Trenholm HL, Cochrane WP, Cohen H, Elliot Jl, Farnworth ER, Friend DW, et al. Survey ofvomitoxin contamination of 1980 Ontario white winter wheat crop: results of survey and feeding trials. J Assoc Off Anal Chem. 1983;66: 92-7.

58. Rotter BA, Thompson BK, Lessard M, Trenholm HL, Tryphonas H. Influence of low-level exposure to fusarium mycotoxins on selected immunological and hematological parameters in young swine. Toxicol Sci. 1994;23:117-24.

59. Arsenakis I, Panzavolta L, Michiels A, Del Pozo Sacristán R, Boyen F, Haesebrouck F, et al. Efficacy of Mycoplasma hyopneumoniae vaccination before and at weaning against experimental challenge infection in pigs. BMC Vet Res. 2016;12:1-7.

60. Jensen CS, Ersbøll AK, Nielsen JP. A meta-analysis comparing the effect of vaccines against Mycoplasma hyopneumoniae on daily weight gain in pigs. Prev Vet Med. 2002;54:265-78.

61. Savard C, Gagnon CA, Chorfi Y. Deoxynivalenol (DON) naturally contaminated feed impairs the immune response induced by porcine reproductive and respiratory syndrome virus (PRRSV) live attenuated vaccine. Vaccine. 2015:33:3881-6.

62. Goyarts T, Dänicke S, Rothkötter HJ, Spilke J, Tiemann U, Schollenberger M. On the effects of a chronic deoxynivalenol intoxication on performance, haematological and serum parameters of pigs when diets are offered either for ad libitum consumption or fed restrictively. J Vet Med A Physiol Pathol Clin Med. 2005;52:305-14.

63. Rotter BA, Thompson BK, Lessard M. Effects of deoxynivalenol-contaminated diet on performance and blood parameters in growing swine. Can J Anim Sci. 1995; 75:297-302.

64. Prelusky DB, Trenholm HL. Tissue distribution of Deoxynivalenol in swine dosed intravenously. J Agric Food Chem. 1991;39:748-51.

65. Blanchard B, Vena MM, Cavalier A, Le Lannic J, Gouranton J, Kobisch M. Electron microscopic observation of the respiratory tract of SPF piglets inoculated with Mycoplasma hyopneumoniae. Vet Microbiol. 1992;30:329-41.

66. Jaques M, Blanchard B, Foiry B, Kobisch M. In vitro colonization of porcine trachea by Mycoplasma hyopneumoniae. Ann Rech Vét, INRA Editions. 1992; 23:239-47.

\section{Ready to submit your research? Choose BMC and benefit from:}

- fast, convenient online submission

- thorough peer review by experienced researchers in your field

- rapid publication on acceptance

- support for research data, including large and complex data types

- gold Open Access which fosters wider collaboration and increased citations

- maximum visibility for your research: over $100 \mathrm{M}$ website views per year

At BMC, research is always in progress.

Learn more biomedcentral.com/submissions 